\title{
Primeros datos sobre vocalización en Leiosaurus catamarcensis (Koslowsky, 1898) y Pristidactylus scapulatus Burmeister, 1861, (Iguania, Leiosauridae) de San Juan, Argentina
}

\author{
First data on vocalization in Leiosaurus catamarcensis (Koslowsky, 1898) \\ and Pristidactylus scapulatus Burmeister, 1861, (Iguania, Leiosauridae) \\ from San Juan, Argentina
}

\author{
Alejandro Laspiur ${ }^{1}$, Eduardo Sanabria $^{2}$ y Juan Carlos Acosta ${ }^{3}$
}

Departamento de Biología e Instituto y Museo de Ciencias Naturales, Facultad de Ciencias Exactas Físicas y Naturales, Universidad Nacional de San Juan.

1laspiursaurus@gmail.com sanabria_eduardo@hotmail.com 3jcacosta@uolsinectis.com.ar

Presentado: $\quad$ 28/03/2006 Aceptado: $\quad$ 05/03/2007

\section{Resumen}

En Argentina no existen antecedentes respecto al estudio de las vocalizaciones en lagartos. Este trabajo pretende dar a conocer las características de la vocalización de dos especies de leiosauridos (Leiosaurus catamarcensis y Pristidactylus scapulatus) con el fin de detectar y evaluar diferencias potencialmente válidas para el uso en sistemática. Las vocalizaciones fueron registradas en condiciones controladas de temperatura y simulando encuentros agresivos. El repertorio observado consistió de dos componentes de vocalización, una de advertencia (ADV) y otra de ataque (AT), diferentes por la longitud temporal de la vocalización. L. catamarcensis y $P$. scapulatus vocalizan en un mismo rango de frecuencia, independientemente del tipo de vocalización. Además encontramos que las vocalizaciones en $L$. catamarcensis son más prolongadas que en $P$. scapulatus.

Palabras clave: Leiosaurus catamarcensis, Pristidactylus scapulatus, vocalizaciones, San Juan

\section{Abstract}

In Argentina not antecedents regarding to lizards vocalization studies exist. This work to seeks to highlight in two leiosaurid species (Leiosaurus catamarcensis and Pristidactylus scapulatus) the vocalization characteristics in order to evaluate potentially valid differences for systematic use. The vocalizations were registered in temperature controlled conditions and simulating aggressive encounters. The observed repertoire consists in two, warning (ADV) and attack (AT) vocalization components, different among themselves due to vocalization temporary length. $L$. catamarcensis and $P$. scapulatus vocalize in a same frequency rank, regardless to the vocalization type. Besides we find that the $L$. catamarcensis vocalizations were more prolongest than $P$. scapulatus.

Keywords: Leiosaurus catamarcensis, Pristidactylus scapulatus, vocalizations, San Juan.

\section{Introducción}

Mientras que muchos lagartos no emiten sonidos, podemos encontrar algunas especies dentro de Lacertia y Crocodilia que vocalizan. Se conoce que al menos seis familias de lagartos, incluido Leiosauridae (=Polychrotidae) (Fouquette com. pers.), Gekkonidae (Tang et al., 2001), Iguanidae, Chamaleonidae, Lacertidae (Frankenberg \& Werner, 1992), Scincidae (Bauer, 2004), que incluyen especies que pueden vocalizar. Sin embargo, pocas han sido estudiadas en profundidad, a excepción de Gekkonidae (Bauer, 2004). Estas vocalizaciones (e.g. Gekko gecko) han sido clasificadas en señales de intimidación, sonidos de estrés y advertencia (Tang et al., 2001), patrones notoriamente etológicos. Sin embargo, las vocalizaciones en reptiles, no se han empleado como diagnóstico para la diferenciación entre especies, como ocurre en otros grupos taxonómicos (Payne, 1986; Sinsch \& Schneider; 1996; Tubaro, 1999; Schneider \& Sinsch. 2006).

En Argentina no existen antecedentes respecto al estudio de las vocalizaciones en reptiles, por lo tanto, este trabajo proporciona información sobre las características de las vocalizaciones de Pristidactylus scapulatus y Leiosaurus catamarcensis.

\section{Material y métodos}

Las vocalizaciones de dos especies de lagartos pertenecientes a la familia Leiosauridae (Frost et al., 2001) fueron estudiadas en condiciones de laboratorio. Los ensayos se realizaron en terrarios de $(60 \times 120 \times 40 \mathrm{~cm}$. $)$ y con individuos aclimatados en un periodo de seis meses. Las vocalizaciones se obtuvieron simulando encuentros agresivos con los ejemplares en forma separada.

Las vocalizaciones se recuperaron con un grabador de audio General Electric de micrófono incorporado. En ambos casos, la temperatura del laboratorio se mantuvo constante $\left(22^{\circ} \mathrm{C}\right)$. Los registros fueron digitalizados y luego analizados con el programa de sonidos Adobe Audition (1.0). Se consideraron las siguientes variables: longitud y frecuencia de vocalización. Los datos se analizaron utilizando estadísticos descriptivos y Análisis de la Varianza (ANOVA) discriminando los tipos de vocalización y las especies consideradas.

Durante tres horas y en forma sucesiva, se registraron 21 vocalizaciones de un ejemplar macho adulto de Leiosaurus catamarcensis (Fig.1), procedente de los Baños de la Laja, Dpto. Albardón (31 19' S, 68 41' W) y 69 vocalizaciones de un ejemplar macho adulto de Pristidactylus scapulatus (Fig.2), procedente de la Reserva Provincial San Guillermo, Dpto. Iglesia, (2857'S; 6846'W).

\section{Resultados}

Encontramos que ambas especies emiten dos tipos de vocalización, diferenciables claramente por la longitud temporal de vocalización (Fig.3a y b; Fig.4a y b), un tipo de vocalización de corta longitud o componente de ataque (AT) y una componente de longitud prolongada o componente de advertencia (ADV). En L. catamarcensis no se encontraron diferencias entre (ADV) y 


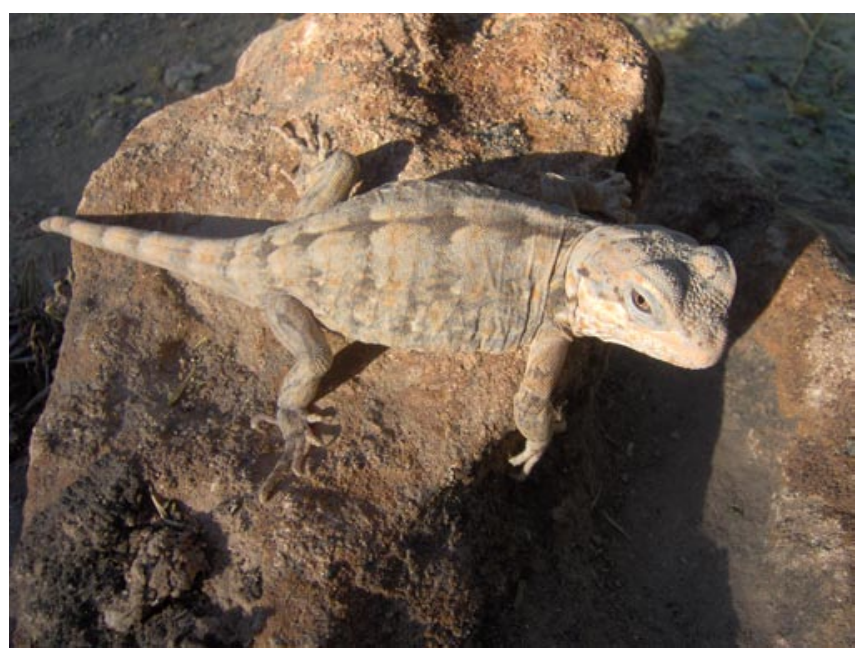

Figura1. Leiosaurus catamarcensis Koslowsky, 1898. Ejemplar macho adulto. Foto: Alejandro Laspiur.

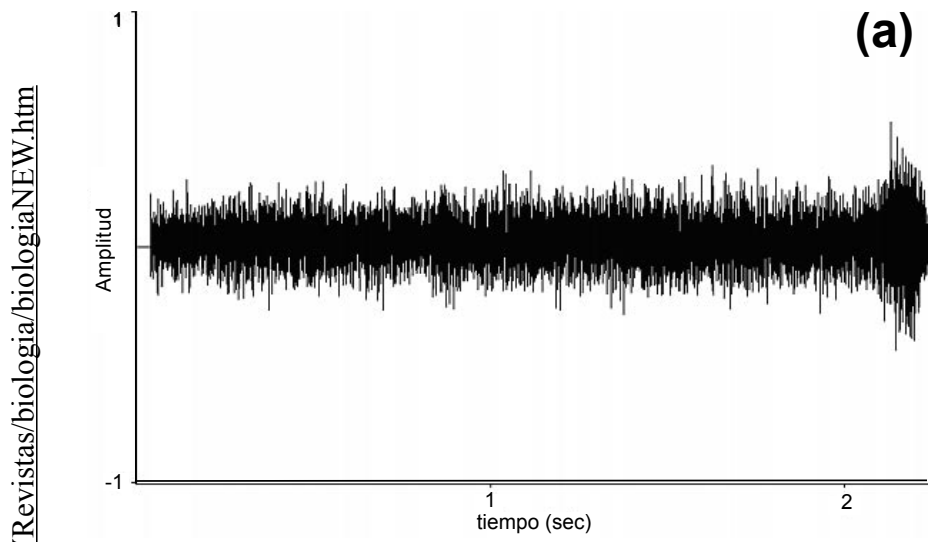

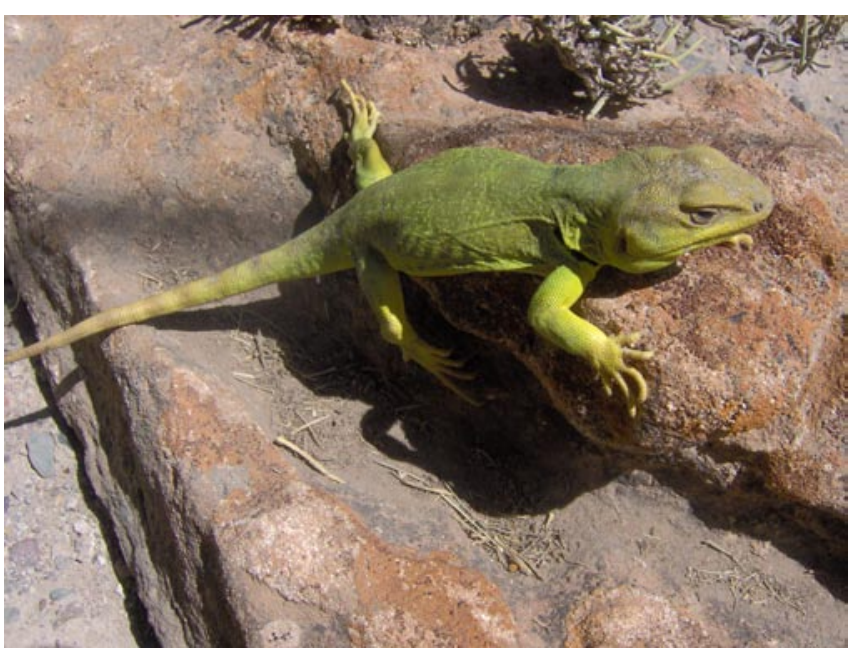

Figura 2. Pristidactylus scapulatus Burmeister, 1861. Ejemplar macho adulto. Foto: Alejandro Laspiur.

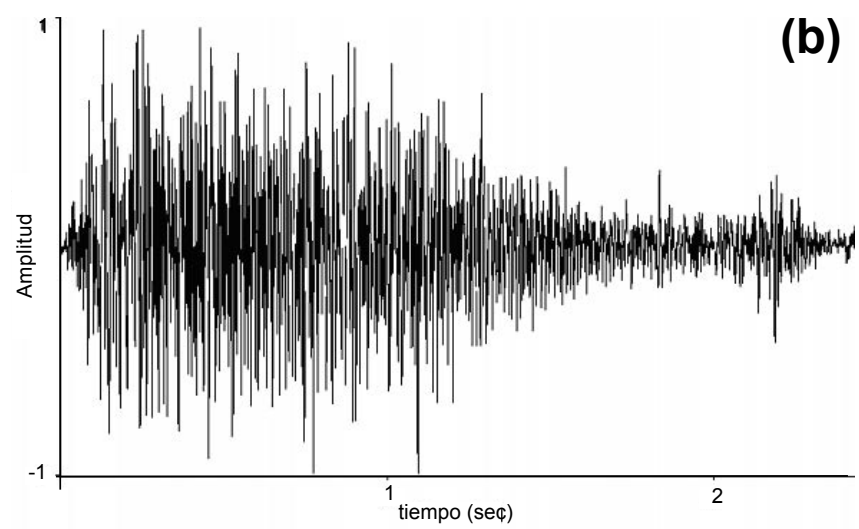

Figura 3. (a) Sonograma de Leiosaurus catamarcensis. Componente de Advertencia (ADV). Longitud prolongada de vocalización. (b) Sonograma de Leiosaurus catamarcensis. Componente de ataque (AT). Longitud breve de vocalización
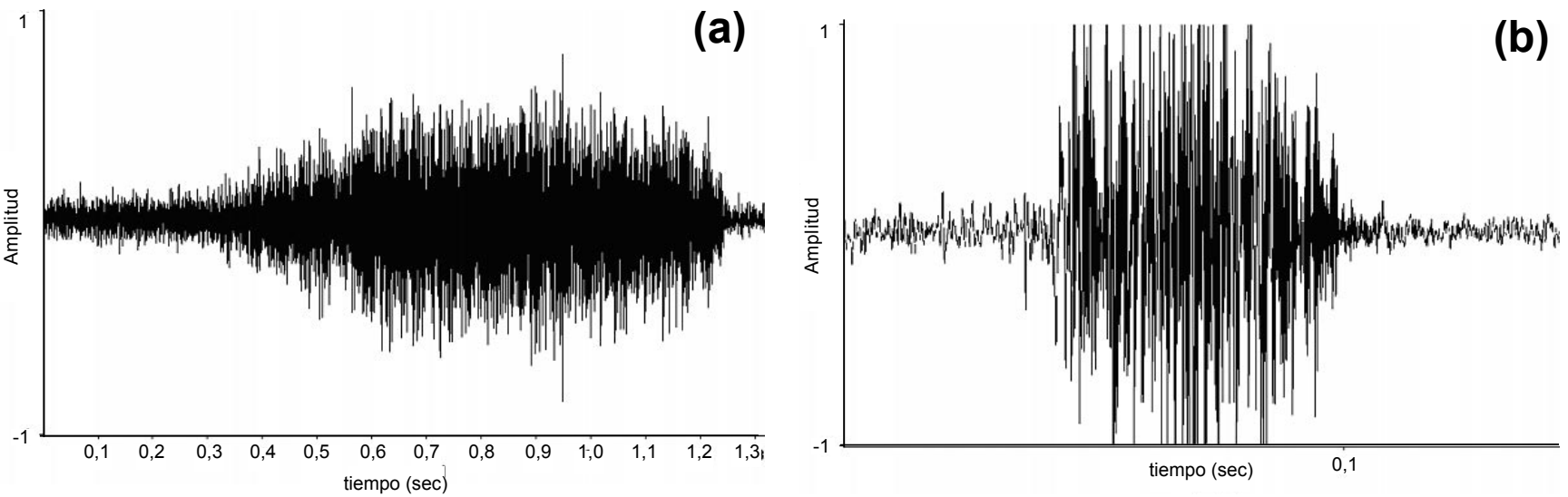

Figura 4. (a) Sonograma de Pristidactylus scapulatus. Componente de advertencia (ADV). Longitud prolongada de vocalización. (b) Sonograma de Pristidactylus scapulatus. Componente de ataque (AT). Longitud breve de vocalización.

(AT) con la frecuencia dominante ( $\mathrm{p}>0,3$; Fig.5); pero si hubieron diferencias entre estas variables y sus longitudes (ANOVA, $\mathrm{F}=$ 19, $\mathrm{p}<0,00001 ; \mathrm{n}=21 ;$ Fig. 6). Para P. scapulatus se encontraron diferencias significativas entre las variables (ADV) y (AT) con respecto a la longitud (ANOVA, $F=67, p<0,00001 ; n=69$; Fig.7). No habiendo diferencias respecto a la frecuencia dominante ( $\mathrm{p}>0,13$; Fig.8). La frecuencia media de la vocalización (ADV) en L. catamarcensis fue de $280,2 \mathrm{~Hz}$. (Tabla 1 ) y la de P. scapulatus fue de $361,4 \mathrm{~Hz}$. (Tabla 1). Estas no fueron significativamente diferentes ( $>0$,85; Fig. 9). Las longitudes de la componente (ADV), se diferenciaron significativamente entre las especies (ANOVA, $\mathrm{F}=41,6 ; \mathrm{p}<0,00001, \mathrm{n}=35$; Fig.10). La frecuencia media de la vocalización del componente de ataque (AT) fue en P. scapulatus fue de $367.4 \mathrm{~Hz}$. (Tabla 2) y en L. catamarcensis de 356.2 Hz. (Tabla 1 y Fig. 9). Estas no fueron significativamente diferentes $(p>0,20)$. La longitud de la componente (AT), fue significativamente disímil entre las especies (ANOVA $\mathrm{F}=33$; $\mathrm{p}<0,00001, \mathrm{n}=55$; Fig. 10). 


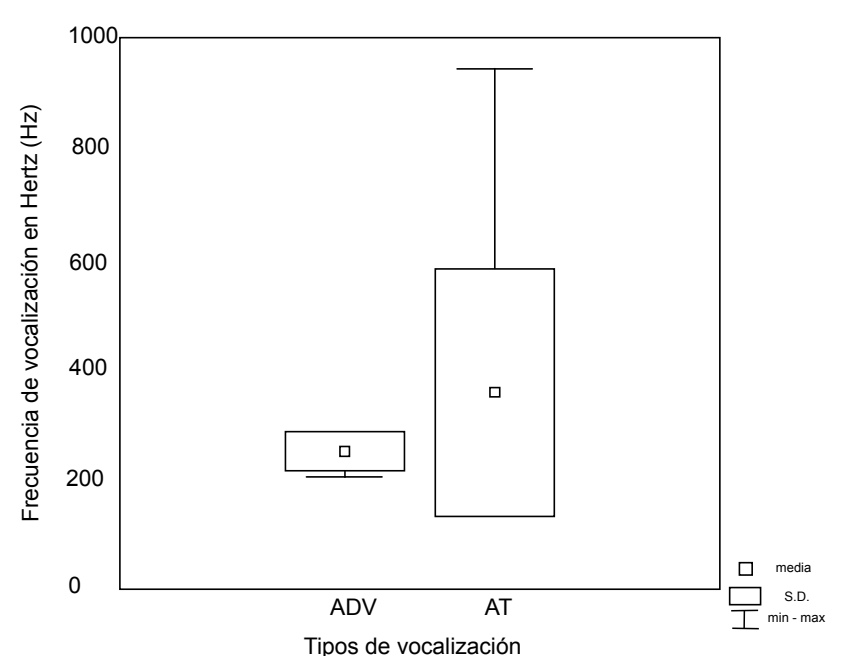

Figura 5. Representación de las diferencias de frecuencia en Hertz $(\mathrm{Hz})$ en las componentes de vocalización en Leiosaurus catamarcensis; ataque (AT); advertencia (ADV).

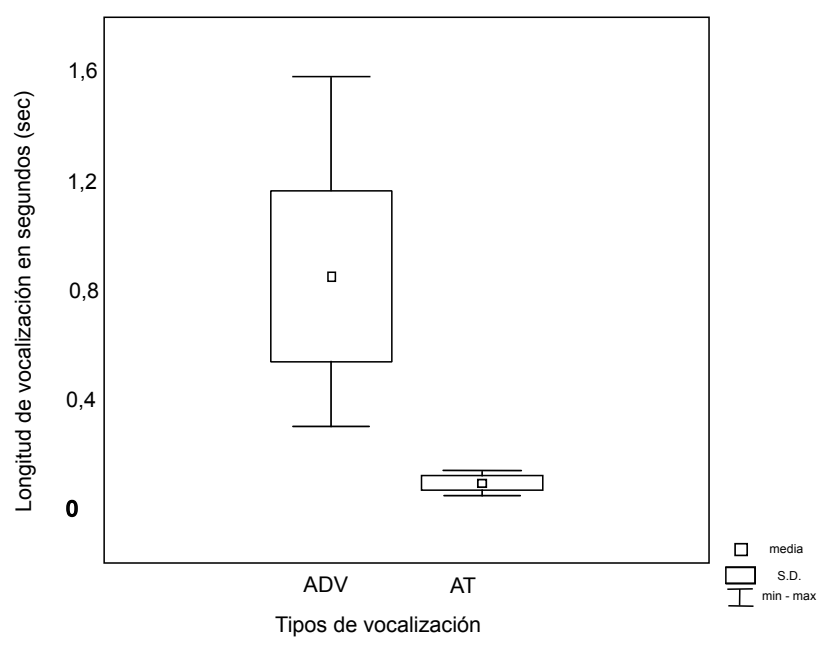

Figura 7. Representación de la diferencias de longitud (seg) en las componentes de vocalización en Pristidactylus scapulatus; ataque (AT); advertencia (ADV).

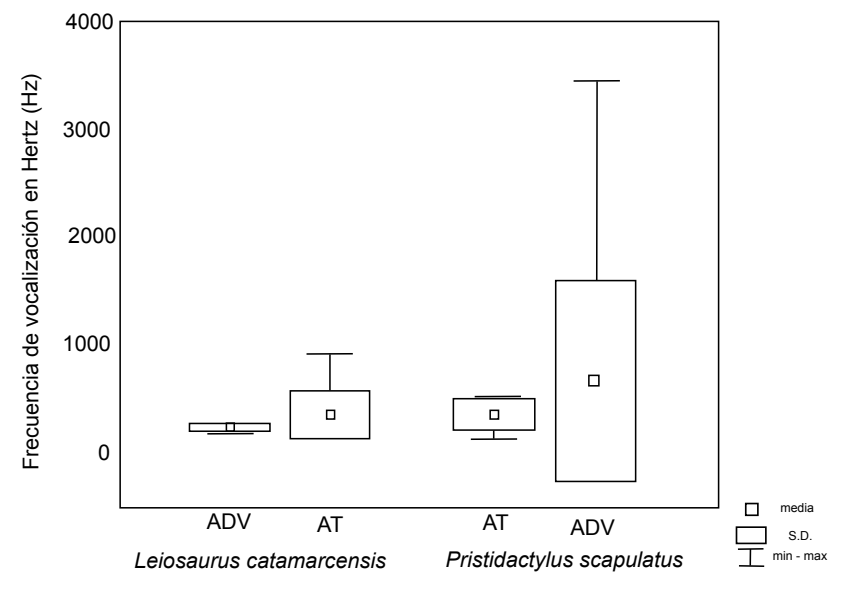

Tipos de vocalización

Figura 9. Representación de la diferencias de frecuencia en Hertz $(\mathrm{Hz})$ en las componentes de vocalización en Leiosaurus catamarcensis y Pristidactylus scapulatus; ataque (AT); advertencia (ADV).

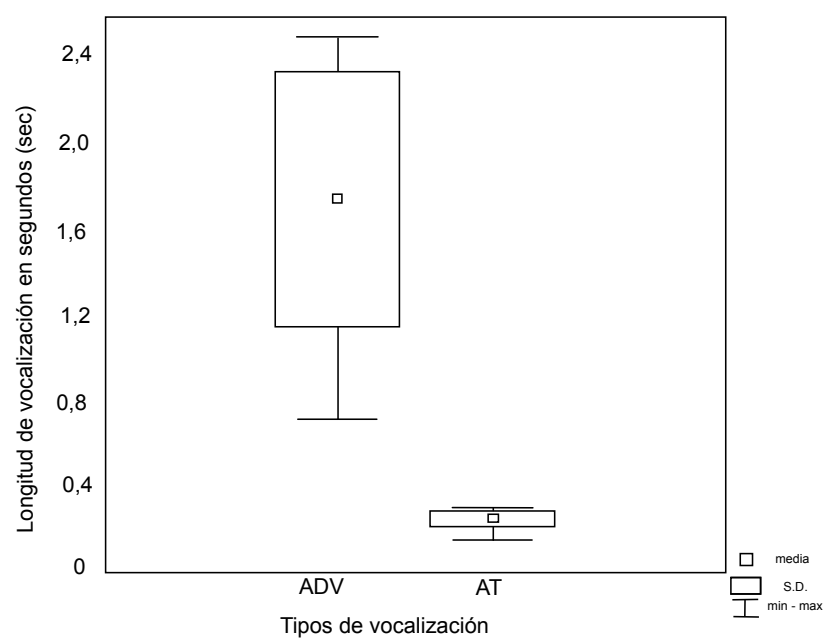

Figura 6. Representación de la diferencias de longitud (seg) en las componentes de vocalización en Leiosaurus catamarcensis; ataque (AT); advertencia (ADV).

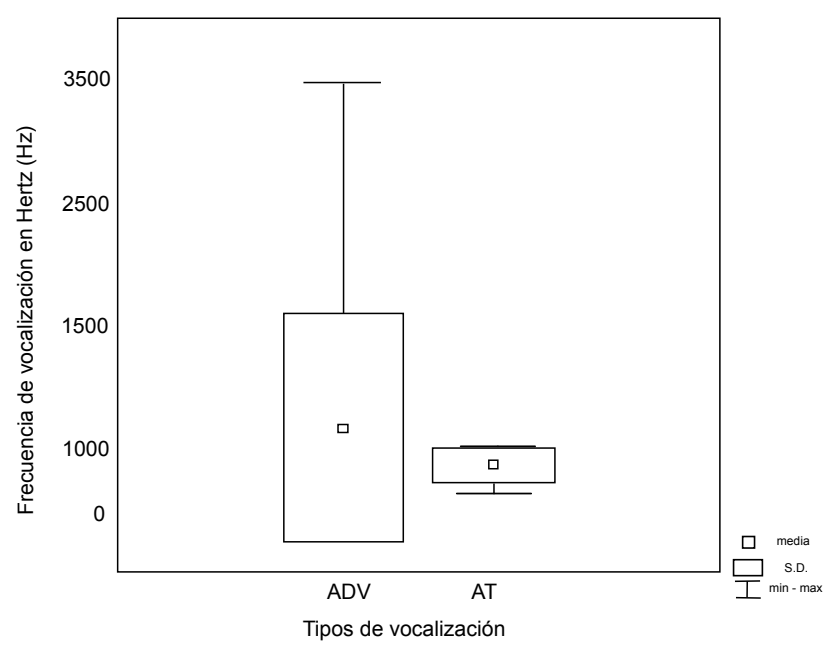

Figura 8. Representación de la diferencias de frecuencia en Hertz $(\mathrm{Hz})$ en las componentes de vocalización en Pristidactylus scapulatus; ataque (AT); advertencia (ADV).

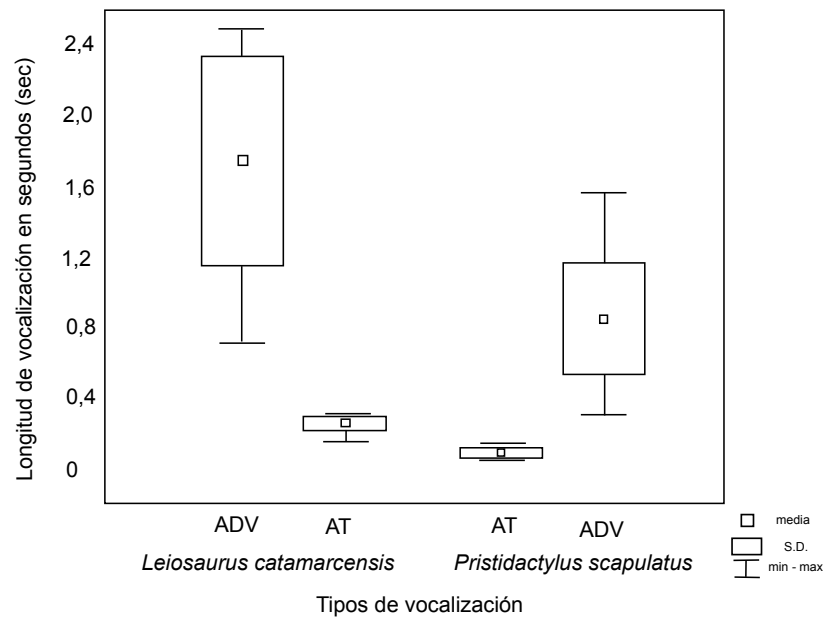

Figura 10. Representación de la diferencias de longitud (seg) en las componentes de vocalización en Leiosaurus catamarcensis y Pristidactylus scapulatus; ataque (AT); advertencia (ADV). 
Tabla 1. Estadística descriptiva de Leiosaurus catamarcensis: ataque (AT), advertencia (ADV). Longitud en segundos (seg), frecuencia en Hertz $(\mathrm{Hz})$, Rango, Media, Desviación Estándar (DE).

\begin{tabular}{ccccc}
\hline Variable & Tipo & Media & Rango & DE \\
\hline Frecuencia (Hz.) & AT & 356,2 & 741,1 & 225,05 \\
Frecuencia (Hz.) & ADV & 280,2 & 82,11 & 36,17 \\
Longitud (seg.) & AT & 0,25 & 0,14 & 0,03 \\
Longitud (seg.) & ADV & 1,47 & 1,78 & 0,59 \\
\hline
\end{tabular}

\section{Discusión}

Encontramos que el repertorio de L. catamarcensis y $P$. scapulatus, consiste en dos componentes de vocalización. Estas vocalizaciones están relacionadas a comportamientos como el de advertencia, en el que se puede observar que el lagarto realiza un desplazamiento breve hacia atrás, acompañado de la erección de la cola. Diferente a la vocalización de ataque, donde el individuo adopta una conducta ofensiva y arremete a morder de forma agresiva. Nos vemos imposibilitados de explicar estos comportamientos fonotácticos, debido a que en condiciones naturales de campo, es posible que estén involucrados factores etológicos que aún no han sido estudiados. Establecemos que L. catamarcensis y $P$. scapulatus vocalizan en un mismo rango de frecuencia, independientemente del tipo de vocalización, diferenciables claramente por la longitud. Además encontramos que las vocalizaciones de $L$. catamarcensis son significativamente más prolongadas temporalmente que las de $P$. scapulatus. Las diferencias observadas permitirían incluir a las vocalizaciones como caracteres en los estudios de la filogenia este grupo.

\section{Agradecimientos}

A la Subsecretaria de Medio Ambiente de la Provincia de San Juan y la Dirección General de Medio Ambiente y Desarrollo Sustentable por otorgar los permisos de captura pertinentes. La realización de este trabajo fue posible por los subsidios otorgados por CICITCA-UNSJ (Res. 053/03-Cs; Proyecto no 21/E333) Proyecto Trianual «Diversidad, Distribución, Bioecología y Conservación de la Herpetofauna de San Juan». En el marco del Programa «Biología, Conservación y Desarrollo de Ecosistemas Áridos y Semiáridos» del Instituto y Museo de Ciencias Naturales- UNSJ.
Tabla 2. Estadística descriptiva de Pristidactylus scapulatus: ataque (AT), advertencia (ADV). Longitud en segundos (seg), frecuencia en Hertz $(\mathrm{Hz})$, Rango, Media, Desviación Estándar (DE).

\begin{tabular}{ccccc}
\hline Variable & Tipo & Media & Rango & DE \\
\hline Frecuencia (Hz.) & AT & 367,4 & 387,9 & 145,8 \\
Frecuencia (Hz.) & ADV & 361,3 & 713,4 & 158 \\
Longitud (seg.) & AT & 0,09 & 0,09 & 0.025 \\
Longitud (seg.) & ADV & 0,82 & 1,27 & 0,31 \\
\hline
\end{tabular}

\section{Literatura citada}

Bauer A. M. 2004. Nannoscincus gracilis (New Caledonian Gracile Dwarf Skink) Vocalization. Herpetological Review 35(3): 268-269.

Frankenberg, E. \& Y. L. Werner. 1992. Vocal communication in the reptilia-facts and questions. Acta Zool. Lilloana 41: 45-62

Frost D. R., R. Etheridge, J. Dani \& T. A. Titus. 2001. Total Evidence, Sequence Alignment, Evolution of Polychrotid Lizards, and a Reclassification of the Iguania (Squamata: Iguania). American Museum Novitates: 1-39.

Payne, R. B. 1986. Bird songs and avian systematics. En: Current Ornithology. Vol. 3. R. J. Johnston, Ed. Plenum Publishing Corporation, New York: 87-126

Tang Y., L. Zhi Zhuang \& Z. Wang Wang. 2001. Advertisement Calls and Their Relation to Reproductive Cycles in Gekko gecko (Reptilia, Lacertilia). Copeia(1): 248-253

Tubaro P. L. 1999. Bioacústica aplicada a la sistemática, conservación y manejo de poblaciones naturales de aves. Etología (7): 19-32.

Sinsch, U. \& H. Schneider. 1996. Bioacustic assesment of the taxonomic status of pool frog populations (Rana lessonae) with reference to a topotylical population. J. Zool. Syst. Evol. Research. 34: 63-73.

Schneider, H. \& U. Sinsch. 2006. Contributions of bioacoustics to the taxonomy of Anura. In: Amphibian Biology. Vol. 7: Phylogeny and Systematics. H. Heatwole \& M. Tyler Eds. Surrey Beatty \& Sons. 